\title{
Recombinant Human Elastase Treatment of Cephalic Veins
}

\section{Marco D Wong1', Karen Bingham², Emma Moss², J Donald Warn ${ }^{3}$, Igor Smirnov¹, Kimberly S Bland', Barry Starcher ${ }^{4}$, F Nicholas Franano ${ }^{1}$} and Steven K Burke ${ }^{1 *}$

${ }^{1}$ Research and Development, Proteon Therapeutics, Inc. Waltham, Massachusetts, USA

${ }^{2}$ Biopta Ltd., Glasgow, Scotland, UK

${ }^{3}$ University of Kansas Medical Center, Kansas City, USA

${ }^{4}$ University of Texas at Tyler, Texas, USA

\begin{abstract}
Background: Vessel injury at the time of Arteriovenous Fistula (AVF) creation may lead to neointimal hyperplasia that impairs AVF maturation. Vonapanitase, a recombinant human chymotrypsin-like elastase family member 1 , is an investigational drug under development to improve AVF maturation and patency. The current studies were designed to document vonapanitase effects in human cephalic veins that are used in AVF creation.

Methods: Human cephalic veins were mounted on a perfusion myograph. Vonapanitase 1.2, 4, 13.2, and 40 $\mu \mathrm{g} / \mathrm{ml}$ or saline was applied drop wise on the vein followed by saline rinse. Vein segments were cut into rings for elastin content determination by desmosine radioimmunoassay and histology. Fluorescently-labelled vonapanitase was applied to veins and adventitial imaging was performed using laser scanning confocal microscopy. In vivo time course experiments were performed by treating rabbit jugular veins and harvesting $1 \mathrm{~h}$ and $4 \mathrm{~h}$ after vonapanitase treatment.

Results / Conclusion: Vonapanitase reduced desmosine content in a dose-related manner. Histology also confirmed a dose-related reduction in elastic fiber staining. Fluorescently-labelled vonapanitase persistently localized to elastic fibers in the vein adventitia. In vivo experiments showed a reduction in desmosine content in jugular veins from $1 \mathrm{~h}$ to $4 \mathrm{~h}$ following treatment. These data suggest that vonapanitase targets elastin in elastic fibers in a dose related manner and that elastase remains in the vessel wall and has catalytic activity for at least $1 \mathrm{~h}$.
\end{abstract}

Keywords: Arteriovenous fistula; Hemodialysis access; Elastase; Elastin; AVF; Vonapanitase; PRT-201; Chymotrypsin-like elastase family member 1 ; CELA1

\section{Abbreviations}

AVF: Arteriovenous Fistula; CELA1: Chymotrypsin-like Elastase family member 1; CKD: Chronic Kidney Disease; PBSP: Phosphate Buffered Saline with 0.01\% Polysorbate 80; PSS: Physiological Saline Solution; RIA Radioimmunoassay (RIA); VVG: Verhoeff Van-Gieson

\section{Introduction}

Vonapanitase (formerly PRT-201), is a recombinant human Chymotrypsin-like Elastase family member 1 (CELA1) and was previously described as type I pancreatic elastase [1]. This novel drug is currently in Phase 3 human clinical trials (PATENCY-1 and PATENCY-2) in CKD patients undergoing AVF placement (clinicaltrials.gov; NCT02110901 and NCT02414841). The goal of the clinical trials is to establish the safety profile of vonapanitase and determine if vonapanitase applied to the outflow vein at the time of AVF creation can improve the success and longevity of AVF surgery.

After vessel injury from AVF surgery there is often the development of neointima hyperplasia from smooth muscle-like cells that migrate to the vessel intima [2]. This neointimal hyperplasia can progress to stenosis or occlusion and cause AVF maturation failure which is a huge clinical problem [2]. When vonapanitase is applied to the outside of surgically exposed cephalic veins immediately after AVF creation it permeates into the tissue where it fragments elastin protein, the major constituent of elastic fibers. Elastic fibers are a major extracellular matrix component of arteries and veins and create a three-dimensional network of fibers that imparts elasticity and determines artery and vein diameters. Complete fragmentation of these elastic fibers increases artery and vein diameters in animals $[3,4]$. On the other hand partial elastin fragmentation generates chemotactic elastin fragments in the adventitia which attract smooth muscle-like cells toward the adventitia, prevents smooth muscle-like cells from migrating towards the intima, and results in a decrease in neointima formation $[5,6]$.

Vonapanitase solution in a volume of $2.5 \mathrm{ml}$ is applied in a single topical administration to the exposed inflow artery, anastomosis, and outflow cephalic vein over 10 minutes followed by a 1 min saline rinse immediately after AVF creation. In a Phase 1 AVF study, vonapanitase at doses of 3.3, 10 and $33 \mu \mathrm{g}$ was associated with a reduction in AVF lumen stenosis and a reduction in patency loss. These benefits were lost at higher vonapanitase doses (100 $\mu \mathrm{g}$ to $9 \mathrm{mg}$ ) [7]. In a Phase $2 \mathrm{AVF}$ study, $10 \mu \mathrm{g}$ and $30 \mu \mathrm{g}$ doses improved AVF maturation (increased AVF blood flow and vein diameter following surgery), prolonged primary and secondary patency, and decreased the number of procedures required to maintain or restore AVF patency [8]. Based on these results Phase $3 \mathrm{AVF}$ trials comparing $30 \mu \mathrm{g}$ versus placebo are on-going (www. clinicaltrials.gov; NCT02110901 and NCT02414841).

The objectives of the experiments conducted were to investigate the pharmacology of the effective vonapanitase doses $(3 \mu \mathrm{g}-33 \mu \mathrm{g})$ on the elastin content of human cephalic vein, the vein most commonly used in the creation of AVFs. This has not been previously reported in the

*Corresponding author: Steven K Burke, Proteon Therapeutics, Inc. 200 West Street Waltham, MA 02451,USA, Tel: 781-890-0102; Fax 781-487-6729; E-mail: sburke@proteontx.com

Received February 29, 2016; Accepted March 31, 2016; Published April 05, 2016

Citation: Wong MD, Bingham K, Moss E, Warn JD, Smirnov I, et al. (2016) Recombinant Human Elastase Treatment of Cephalic Veins. Cardiovasc Pharm Open Access 5: 178. doi:10.4176/2329-6607.1000178

Copyright: ( 92016 Wong MD, et al. This is an open-access article distributed under the terms of the Creative Commons Attribution License, which permits unrestricted use, distribution, and reproduction in any medium, provided the original author and source are credited. 
literature. Human cephalic veins obtained from recently deceased donors were mounted in a perfusion myograph device which can maintain a set transmural pressure and impart stretch on the vein tissue to simulate in vivo conditions [9]. Previous work has shown that stretch on elastic tissue increases the sensitivity of the elastic fibers to elastase [10,11]. To mimic the pressure condition of a vein in an AVF, the vein was pressurized to 25 $\mathrm{mm} \mathrm{Hg}$ and $50 \mathrm{~mm} \mathrm{Hg}$, which is the range of pressures experienced by a vein in a typical AVF [12]. Vonapanitase was also fluorescently labelled to identify vonapanitase in the blood vessel wall. In vivo time course experiments were performed to determine how long vonapanitase was catalytically active in the blood vessel wall.

Elastin content was measured by desmosine content. Desmosine is a protein cross-link unique to elastin that was quantitated using a Radioimmunoassay (RIA) [13]. Histology was performed using Verhoeff-Van Gieson (VVG) or Movat's Pentachrome that stain elastic fibers blue or black.

\section{Methods}

\section{Vein harvest}

For the myography experiment, the veins were provided by the National Disease Research Interchange (NDRI). The left and right cephalic veins were retrieved from the upper and lower arms of deceased donors within $24 \mathrm{~h}$ of death, placed in ice-cold Physiological Saline Solution (PSS) consisting of $119.0 \mathrm{mM} \mathrm{NaCl}, 4.7 \mathrm{mM} \mathrm{KCl}$, $1.2 \mathrm{mM} \mathrm{MgSO}_{4}, 24.9 \mathrm{mM} \mathrm{NaHCO}_{3}, 1.2 \mathrm{mM} \mathrm{KH}_{2} \mathrm{PO}_{4}, 2.5 \mathrm{mM} \mathrm{CaCl}$ and $11.1 \mathrm{mM}$ glucose prior to transport by a courier. The veins were used for experiments immediately upon receipt. For the fluorescence experiment, the human cephalic veins from recently deceased donors were provided by the International Institute for the Advancement of Medicine (IIAM). All donors or their families signed a consent form that specified that the tissues could be used for research.

\section{Myography and treatment}

Cephalic veins were cut to approximately $2.5 \mathrm{~cm}$ and mounted with silk sutures on the stainless steel cannula of a perfusion myograph (PM-1, Biopta Ltd, Glasgow, Scotland) [9]. PSS was added to the chamber and also perfused through the vein lumen. The intraluminal pressure was set to $40 \mathrm{~mm} \mathrm{Hg}$ and equilibrated for $5 \mathrm{~min}$. During the ten minute treatment period, the pressure was set to $25 \mathrm{~mm} \mathrm{Hg}$ for 5 minutes and then to $50 \mathrm{~mm} \mathrm{Hg}$ for 5 minutes to simulate the pressure and stretch experienced by the vein during the cardiac cycle. $2.5 \mathrm{ml}$ of dosing solution was applied as drops to the vein every $30 \mathrm{sec}$ using a micropipette. Four doses of vonapanitase in phosphate buffered saline pH 7.4 containing $0.01 \%$ Polysorbate 80 (PBSP) were used: $3,10,33$ and $100 \mu \mathrm{g}(2.5 \mathrm{ml}$ of $1.2,4,13.2$, and $40 \mu \mathrm{g} / \mathrm{ml}$ solutions, respectively). Control veins were treated with PBSP. Following treatment, the vein was rinsed by filling then draining the bath chamber three times with PSS.

\section{Measurements}

The $2.5 \mathrm{~cm}$ cephalic vein segment was removed from the myograph and the ends used to attach the vein to the myograph were trimmed away. The vein was stored in PSS for $3 \mathrm{~h}$ at room temperature then at $4^{\circ} \mathrm{C}$ overnight. The next day the vein was placed in $10 \%$ buffered formalin and was stored at room temperature overnight, after which the vein was cut into rings $2 \mathrm{~mm}-3 \mathrm{~mm}$ in length. The rings were used for desmosine determination and histology.For desmosine determination, the vein rings were hydrolyzed with $6 \mathrm{~N} \mathrm{HCl}$ then dried in a speed vacuum and suspended with Nanopure water to a concentration of $1 \mathrm{mg} / \mathrm{ml}$. Samples were analyzed by desmosine RIA to determine the amount of elastin remaining [13]. For histology, the rings were embedded in paraffin, sectioned at a thickness of approximately $5 \mu \mathrm{m}$, and stained with VVG or Movat's Pentachrome.

\section{Fluorescence}

Fluorescently-labeled vonapanitase was created with a Dylight labeling kit (Pierce, Rockford, IL) using a protocol developed for labeling porcine CELA1 [11]. $0.1 \mathrm{mg}$ of vonapanitase in PBSP $(0.1 \mathrm{ml}$ of $1 \mathrm{mg} /$ $\mathrm{ml}$ ) was added to the Dylight Reagent containing a $633 \mathrm{~nm}$ fluorophore and vortexed gently. The mixture was incubated for $60 \mathrm{~min}$ at room temperature and protected from light. The labeling reaction was placed in a spin column with purification resin and centrifuged to collect fluorescently-labeled vonapanitase. A $2.5 \mathrm{~cm}$ segment of cephalic vein was mounted in a perfusion myograph (Danish Myo Technologies, Aarhus, Denmark) and the pressure was cycled between $25 \mathrm{~mm} \mathrm{Hg}$ and $50 \mathrm{~mm} \mathrm{Hg}$ every $1 \mathrm{~min}$ while $2.5 \mathrm{ml}$ of $12 \mu \mathrm{g} / \mathrm{ml}$ of fluorescentlylabelled vonapanitase was placed on the vein drop wise over $10 \mathrm{~min}$ using an angiocatheter. After treatment the vein was rinsed with saline for $1 \mathrm{~min}$. Another $2.5 \mathrm{~cm}$ segment of vein was treated similarly with PBSP only. Adventitial imaging was performed to visualize elastic fiber autofluorescence at $488 \mathrm{~nm}$ and fluorescently-labeled vonapanitase at $638 \mathrm{~nm}$ using laser scanning confocal microscopy (LSCM-Nikon Corporation, Mellville NY; Nikon 90iupright, far red 638 diode laser with 650 long pass filter, 488 multiline argon laser with 515 / 30 detector, visualizing in separate excitation / emission detection passes). Elastin is known to be autofluorescent [11]. A PBSP treated vessel was scanned and then a vonapanitase treated vessel was scanned. Scans were done beginning at the outer aspect of the adventitia in $0.5 \mu \mathrm{m}$ section steps down deeper into the adventitia up to $80 \mu \mathrm{m}$ in 160 steps, scanning a $647 \mu \mathrm{m}$ square area.

\section{In-vivo time course}

All animal procedures were performed in accordance with policies and procedures of the Office for Laboratory Animal Welfare and in compliance with the Guide for the Care and Use of Laboratory Animals. The procedures were reviewed and approved by Institutional Animal Care and Use Committee (IACUC) of Kansas University Medical Center which is fully accredited by the Association for the Assessment of Accreditation of Laboratory Animal Care, International (AAALAC).

Two New Zealand White rabbits ( $4 \mathrm{Kg}-4.5 \mathrm{Kg}$ ) were used for these experiments. Each rabbit was administered general anesthesia with isoflurane in the operating room. A midline neck incision was made and rabbit external jugular veins were exposed bilaterally. For the first rabbit the left external jugular vein was treated drop wise with $2.5 \mathrm{ml}$ of $4 \mu \mathrm{g} / \mathrm{ml}$ vonapanitase for $10 \mathrm{~min}$ followed by a $1 \mathrm{~min}$ saline rinse. After 3 hours the contralateral external jugular vein was treated drop wise with $2.5 \mathrm{ml}$ of $4 \mu \mathrm{g} / \mathrm{ml}$ vonapanitase for $10 \mathrm{~min}$ followed by a $1 \mathrm{~min}$ saline rinse. For the second rabbit a similar procedure was performed but the right external jugular vein was treated first. One hour after the second treatment, the rabbits were euthanized with Beuthanasia (a mixture of phenytoin and pentobarbital) $100 \mathrm{mg} / \mathrm{kg}$ IV and both external jugular veins for each rabbit were harvested and then fixed with $10 \%$ buffered formalin. The harvested external jugular veins were hydrolyzed with 6 $\mathrm{N} \mathrm{HCl}$ and analyzed by desmosine RIA with 10 replicates to determine elastin content.

\section{Results}

Human cephalic veins were harvested from 4 donors for the perfusion myography experiments. In 1 donor the left arm cephalic vein was used, in 2 donors the right arm cephalic veins were used, and in 1 donor both the left and right arm cephalic veins were used. In the 
case of two donors, there was insufficient vein to complete testing at each vonapanitase concentration. Therefore there were 5 replicates for PBSP and 4 replicates for each of the vonapanitase concentrations tested. Table 1 summarizes the mean desmosine content of vein treated with various concentrations of vonapanitase or PBSP. Figure 1 displays the mean percentage reductions in desmosine content relative to PBSP treatment as a function of vonapanitase concentration ranging from essentially no reduction at the $1.2 \mu \mathrm{g} / \mathrm{ml}$ concentration to $80 \%$ reduction at the $40 \mu \mathrm{g} / \mathrm{ml}$ concentration. There was a significant $(\mathrm{p}<0.05)$ relationship between log vonapanitase concentration and desmosine content using linear regression.

\section{Histology}

Figure 2 displays images of untreated cephalic vein stained with (a) Verhoeff Van-Gieson (VVG) and (B) Movat's Pentachrome stains. Black elastic fibers were evident in the adventitia, media, and intima with both stains. Staining of cephalic vein treated with $1.2 \mu \mathrm{g} /$ $\mathrm{ml}$ vonapanitase was similar to that of vein treated with PBSP. Vein treated with 4 and $13.2 \mu \mathrm{g} / \mathrm{ml}$ vonapanitase had elastic fibers present but clearly reduced relative to PBSP treated vein (data not shown). Figure 3 displays representative images of human cephalic vein treated with PBSP (A) or the highest concentration $(40 \mu \mathrm{g} / \mathrm{ml})$ of vonapanitase (B) using the VVG stain. There was substantially less elastic fiber staining in the $40 \mu \mathrm{g} / \mathrm{ml}$ vonapanitase treated section compared to the PBSP treated section as evidenced by an absence of black elastic fibers in the image in Figure 3 panel B. In the $40 \mu \mathrm{g} / \mathrm{ml}$ vonapanitase treated section, elastic fiber staining was absent from the adventitia, whereas in some sections residual staining of elastic fibers were present in the media and intima.

\section{Fluorescence}

Figure 4 displays the laser scanning confocal microscopy fluorescent images of the vein adventitia. Panel A demonstrates green elastic fiber auto-fluorescence. Panel B demonstrates blue fluorescently-labeled vonapanitase in a fibrillar pattern consistent with elastic fiber colocalization indicating that after the saline wash vonapanitase was still present and co-localized with elastic fibers in the outer aspect of the adventitia.

\section{In-vivo time course}

In the first rabbit, desmosine content was 352.9 picomoles of desmosine $(\mathrm{pmD}) / \mathrm{mg}$ protein $(\mathrm{mgP})$ in the right jugular vein $1 \mathrm{~h}$ following treatment and $233.7 \mathrm{pmD} / \mathrm{mgP}$ in the left jugular vein $4 \mathrm{~h}$ following treatment. In the second rabbit desmosine content was 580.5 $\mathrm{pmD} / \mathrm{mgP}$ in the left jugular vein $1 \mathrm{~h}$ following treatment and 433.9 $\mathrm{pmD} / \mathrm{mgP}$ in the right jugular vein $4 \mathrm{~h}$ following treatment. Veins treated with vonapanitase for $4 \mathrm{~h}$ had $25 \%$ - 30\% less elastin content than veins treated for $1 \mathrm{~h}$ indicating ongoing elastase activity.

\section{Discussion}

The purpose of this study was to define the vonapanitase dose-response curve for elastic fiber removal and to gain a better understanding of the mechanism of action of microgram doses of vonapanitase on human cephalic veins used in the creation of AVFs. Treatments were administered in a manner that mimicked the human dosing in clinical trials including the $10 \mathrm{~min}$ application time, saline wash, and use of pressure and stretch on the vein during treatment. In the current experiments, vonapanitase removed elastic fibers from the cephalic vein in a dose-related manner as assessed by both desmosine and histological staining of elastic fibers. These two methods of detecting elastin fibers are complementary. Desmosine RIA analysis is quantitative for elastin, the major component of the elastic fiber [13]. Standard histology is not sensitive enough to show small changes in elastic fiber staining but the histological images are capable of conveying the pattern of elastic fiber removal [14]. Both VVG and Movat's Pentachrome readily stained elastic fibers which were easy to distinguish from surrounding tissue. The greatest effect on elastic fiber

\begin{tabular}{|c|c|c|c|c|c|}
\hline Concentration & Dose & N & Mean & SD & $\begin{array}{c}\text { P-Value } \\
\text { Vs. } \\
\text { Placebo }\end{array}$ \\
\hline 0 (PBSP control) & $0 \mathrm{mg}$ & 5 & 863 & 368 & \\
\hline $1.2 \mu \mathrm{g} / \mathrm{ml}$ & $3 \mu \mathrm{g}$ & 4 & 840 & 442 & 0.76 \\
\hline $4 \mu \mathrm{g} / \mathrm{ml}$ & $10 \mu \mathrm{g}$ & 4 & 779 & 251 & 0.07 \\
\hline $13.2 \mu \mathrm{g} / \mathrm{ml}$ & $33 \mu \mathrm{g}$ & 4 & 492 & 573 & 0.07 \\
\hline $40 \mu \mathrm{g} / \mathrm{ml}$ & $100 \mu \mathrm{g}$ & 4 & 258 & 340 & $<0.001$ \\
\hline
\end{tabular}

${ }^{1}$ T-test

Table 1: Mean desmosine content of cephalic arm veins treated with PBSP or varying concentrations of vonapanitase.

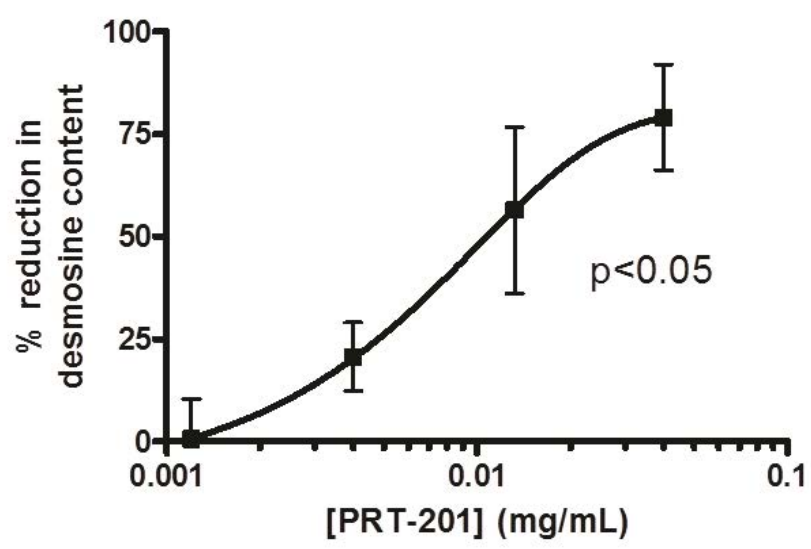

Figure 1: Mean percentage reduction in desmosine content of human cephalic arm veins treated with varying concentrations of vonapanitase relative to PBSP treatment.
(A)

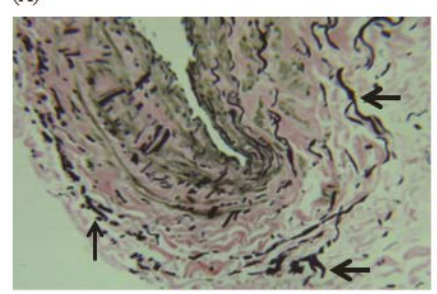

(B)

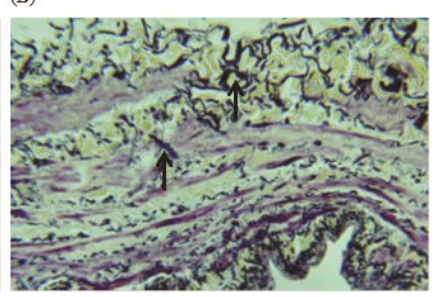

Figure 2: Cephalic vein treated with PBSP and stained using VVG stain (A) or Movat's Pentachrome Stain (B). (40X magnification) Black fibers (arrows) are elastic fibers.
(A)

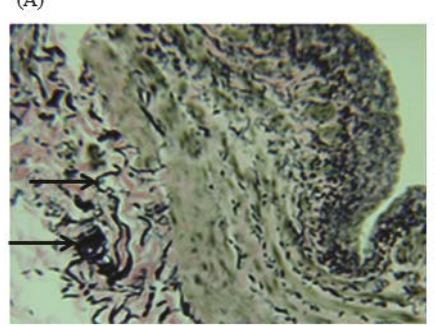

(B)

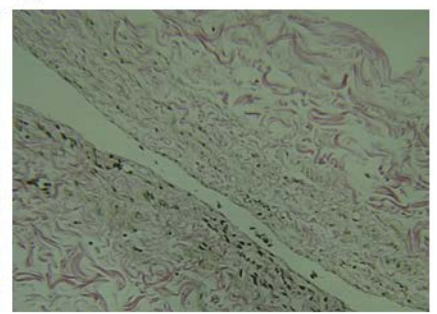

Figure 3: Cephalic veins treated with PBSP (A) and $40 \mu \mathrm{g} / \mathrm{ml}$ vonapanitase and stained using VVG stain. (40X magnification). Black fibers (arrows) are elastic fibers. 
(A)

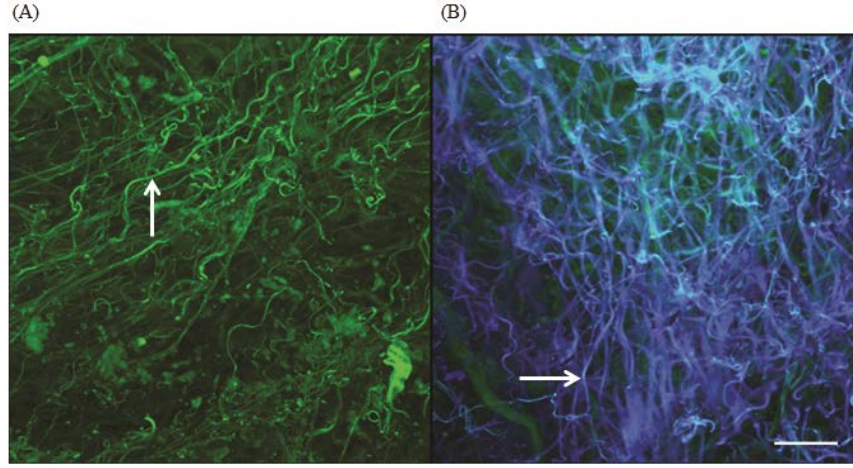

Figure 4: Cephalic vein treated with $\operatorname{PBSP}(\mathrm{A})$ and fluorescently-labelled vonapanitase (B) Arrows indicate elastic fibers. Scale bar denotes $100 \mathrm{um}$.

removal was observed in the vein adventitia where vonapanitase was applied. This is consistent with the goal of generating elastin peptide fragments in the adventitia. These fragments are recognized by cells that are believed to participate in the formation of intimal hyperplasia and provide chemotactic signals that decrease the migration of the cell types from the adventitia to the intima $[5,6]$. We had previously used other models to define the vonapanitase dose- response curve but each had limitations. A rabbit AVF model was confounded by the thinness of rabbit vein wall relative to the human vein wall. Vessel wall thickness correlates directly with the dose of vonapanitase required to remove elastic fibers [15]. Treatment of isolated human artery and vein rings failed to account for vessel pressure and stretch which have a significant impact on elastic fiber sensitivity to elastase [10,11]. Stretch appears to expand the elastic fiber network exposing elastase binding sites and altering binding kinetics. Caution is needed when relying on data obtained from in-vitro experiments with unstretched artery and vein rings in order to estimate the potency of vonapanitase for elastin removal in-vivo (unpublished results).

In an AVF clinical trial, doses of 3, 10, and 33 microgram doses were more effective than doses of $100 \mu \mathrm{g}$ and higher in preventing AVF stenosis and prolonging AVF patency $[7,8]$. In the current study, a 100 microgram dose removed nearly all the elastin from the cephalic vein and there was histological evidence of elastin fragmentation in the venous media and intima. This may be too high a dose since generating elastin fragments throughout the vessel wall may not result in a chemotactic gradient of fragments necessary to retain cells in the adventitia $[5,6]$.

In order to understand how a 10 min application of microgram doses of vonapanitase followed by washing could result in a substantial elastic fiber fragmentation; we conducted studies using fluorescentlylabeled vonapanitase applied for $10 \mathrm{~min}$ followed by a $1 \mathrm{~min}$ saline wash. Fluorescent vonapanitase was seen in a fibrillar pattern in the outer layer of the adventitia of the cephalic vein consistent with elastic fiber binding. The vonapanitase remaining in the vein was shown to be catalytically active in vivo in the time course studies conducted in rabbits. Vein elastin content declined by $25 \%$ - 30\% between $1 \mathrm{~h}$ and $4 \mathrm{~h}$ following treatment. Further time course experiments would need to be performed to determine exactly how long vonapanitase is catalytically active in the adventitia. Vonapanitase is inactivated by antiproteases alpha 1-antitrypsin and alpha 2-macroglobulin present in blood [16] but the vasa vasorum is likely disrupted during creation of the AVF and it is unclear whether antiproteases have ready access to the vein adventitia in this setting.

In conclusion, these studies provided evidence that vonapanitase doses as low as $10 \mu \mathrm{g}$ and $33 \mu \mathrm{g}$ applied topically over $10 \mathrm{~min}$ followed by a saline wash localized vonapanitase to the outer aspect of the adventitia where it had catalytic activity for at least $1 \mathrm{~h}$ and resulted in measureable elastic fiber removal. The $100 \mu \mathrm{g}$ dose removed almost all of the elastic fibers from the vein and possibly would not form a chemotactic elastin fragment concentration gradient. These findings provide a rationale for why doses of 10 to 33 micrograms would have a beneficial effect on AVFs and why the benefit is lost at higher doses.

\section{Acknowledgement}

This study was funded by Proteon Therapeutics, Inc. The human veins used in this study were provided by the National Disease Research Interchange (NDRI) with support by grant number 5 U42 RR006042 from NIH and from the International Institute for the Advancement of Medicine (IIAM). The authors thank Dr. Colin Russell, PDT Manufacturing Ltd., Scotland, UK, for overseeing the histology.

\section{References}

1. Kawashima I, Tani T, Mita-Honjo K, Shimoda-Takano K, Ohmine T, et al (1992) Genomic organization of the human homologue of the rat pancreatic elastase I gene. DNA Seq 2: 303-312.

2. Roy-Chaudhury P, Arend L, Zhang J, Krishnamoorthy M, Wang Y, et al. (2007) Neointimal hyperplasia in early arteriovenous fistula failure. Am J Kidney Dis 50: 782-790.

3. Dobrin PB, Baker WH, Gley WC (1984) Elastolytic and collagenolytic studies of arteries. Implications for the mechanical properties of aneurysms. Arch Surg 119: $405-409$

4. Kitoh T, Kawai Y, Ohhashi T (1993) Effects of collagenase, elastase, and hyaluronidase on mechanical properties of isolated dog jugular veins. Am J Physiol 265: H273-280.

5. Amabile PG, Wong H, Uy M, Boroumand S, Elkins CJ, et al. (2002) In vivo vascular engineering of vein grafts: Directed migration of smooth muscle cells by perivascular release of elastase limits neointimal proliferation. J Vasc Interv Radiol 13: 709-715.

6. Wong AH, Waugh JM, Amabile PG, Yuksel E, Dake MD (2002) In vivo vascula engineering: directed migration of smooth muscle cells to limit neointima. Tissue Eng 8: 189-199.

7. Peden EK, Leeser DB, Dixon BS, El-Khatib MT, Roy-Chaudhury P, et al. (2013) A multi-center, dose-escalation study of human type I pancreatic elastase (PRT201) administered after arteriovenous fistula creation. J Vasc Access 14: 143-151.

8. Hye RJ, Peden EK, O'Connor TP, Browne BJ, Dixon BS, et al. (2014) Human type I pancreatic elastase treatment of arteriovenous fistulas in patients with chronic kidney disease. J Vasc Surg 60: 454-461.

9. Moss E, Lynagh S, Smith D, Kelly S, McDaid A, et al. (2010) A novel system for the investigation of microvascular dysfunction including vascular permeability and flow-mediated dilatation in pressurised human arteries. J Pharmacol Toxicol Methods 62: 40-46.

10. Suki B, Jesudason R, Sato S, Parameswaran H, Araujo AD, et al. (2012) Mechanical failure, stress redistribution, elastase activity and binding site availability on elastin during the progression of emphysema. Pulm Pharmacol Ther 25: 268-275.

11. Jesudason R, Sato S, Parameswaran H, Araujo AD, Majumdar A, et al. (2010) Mechanical forces regulate elastase activity and binding site availability in lung elastin. Biophys J 99: 3076-3083.

12. Corpataux JM, Haesler E, Silacci P, Ris HB, Hayoz D (2002) Low-pressure environment and remodelling of the forearm vein in Brescia-Cimino haemodialysis access. Nephrol Dial Transplant 17: 1057-1062.

13. Starcher BC, Mecham RP (1981) Desmosine radioimmunoassay as a means of studying elastogenesis in cell culture. Connect Tissue Res 8: 255-258.

14. Masuda H, Zhuang YJ, Singh TM, Kawamura K, Murakami M, et al. (1999) Adaptive remodeling of internal elastic lamina and endothelial lining during flowinduced arterial enlargement. Arterioscler Thromb Vasc Biol 19: 2298-2307.

15. Burke SK, Franano FN, LaRochelle A, Mendenhall HV (2008) Local application of recombinant human type I pancreatic elastase (PRT-201) to an arteriovenous fistula (AVF) increases AVF blood flow in a rabbit model. J Am Soc Nephrol 19 252-253A.

16. Qamar AA, Burke SK, Lafleur JD, Ding BC, Bland KS, et al. (2012) The ability of serum from alpha 1-antitrypsin-deficient patients to inhibit PRT-201, a recombinant human type I pancreatic elastase. Biotechnol Appl Biochem 59: 22-28. 